\title{
Aspectos facilitadores e dificultadores do trabalho do enfermeiro em cargos gerenciais no âmbito hospitalar
}

Aspectos que facilitan y dificultan el trabajo del enfermero en puestos de gestión hospitalaria

Facilitating and hindering aspects of nurses' work in hospital managerial positions

\author{
Nilce Mara da Silva ${ }^{\mathrm{I}}$, Vivian Aline Mininel ${ }^{\mathrm{II}}$, Silvia Helena Henriques ${ }^{\mathrm{III}}$ \\ Ana Martha de Almeida Limongelli ${ }^{\mathrm{Iv}}$, Ana Paula Pereirav ${ }^{\mathrm{v}}$, Lucieli Dias Pedreschi Chaves ${ }^{\mathrm{VI}}$
}

\begin{abstract}
Resumo: Objetivo: identificar os aspectos que facilitam e dificultam o trabalho do enfermeiro em cargos gerenciais em um hospital público de ensino. Método: estudo descritivo, de abordagem qualitativa, desenvolvido em hospital público terciário, utilizando a Técnica do Incidente Crítico. A amostra foi constituída por 15 enfermeiros em cargos gerenciais há, pelo menos, um ano. Resultados: foram analisadas 42 situações, sendo 66,6\% de referências negativas; 57 comportamentos apresentaram $84,2 \%$ de referências positivas e 74 consequências com $58,1 \%$ de referências negativas. Conclusão: os aspectos que facilitam o trabalho do enfermeiro em cargos gerenciais são a situações/comportamentos/consequências referentes à interação da equipe/paciente/família; gerenciamento da unidade de trabalho, a implantação do grupo gestor e a comunicação. Em contrapartida, os aspectos dificultadores estão relacionados à estrutura organizacional, gestão de infraestrutura e gestão de pessoas.
\end{abstract}

Descritores: Enfermagem; Organização e administração; Hospitais públicos; Administração hospitalar

\begin{abstract}
Objective: identify aspects that facilitate and hinder the work of nurses in managerial positions in a public teaching hospital. Method: descriptive study with a qualitative approach, developed in a public, tertiary hospital, using the Critical Incident Technique. Sample consisting of 15 nurses in managerial positions for at least one year. Results: 42 situations were obtained, being $66.6 \%$ of negative references; 57 behaviors showing $84.2 \%$ positive references and 74 consequences getting $58.1 \%$ negative references. Conclusion: The aspects that facilitate the work of nurses in managerial positions are situations / behaviors / consequences related to team / patient /
\end{abstract}

\footnotetext{
I Enfermeira. Hospital das Clinicas da Faculdade de Medicina de Ribeirão Preto/SP- Brasil. Núcleo Interno de Regulação. Email: nilcemara.silva@hotmail.com ORCID iD http://orcid.org/0000-0002-3989-9282

II Enfermeira Profa. Dra. Departamento de Enfermagem do Centro de Ciências Biológicas e da Saúde da Universidade Federal de São Carlos UFSCAR; São Carlos SP, Brasil. E-mail: vivian.aline@gmail.com ORCID iD http://orcid.org/0000-0001-9985-5575

III Enfermeira Profa. Dra. Escola de Enfermagem de Ribeirão Preto, Universidade de São Paulo (USP), SP, Brasil. E-mail shcamelo@eerp.usp.br ORCID iD http://orcid.org/0000-0003-2089-3304

IV Professora Dra. em Educação: Claretiano centro universitário: Batatais, São Paulo, Brasil. E-mail anamarta.claretiano@gmail.com; ORCID iD.org 0000-00016362-7124

${ }^{\vee}$ Enfermeira. Centro de referência em Saúde da Mulher - Mater Ribeirão Preto, São Paulo, Brasil. E-mail: appereira@hcrp.usp.br ORCID iD http://orcid.org/0000-0002-6373-4162

VI Enfermeira Profa. Dra. Escola de Enfermagem de Ribeirão Preto, Universidade de São Paulo (USP), Brasil. E-mail: dpchaves@eerp.usp.br ORCID iD http://orcid.org/0000-0002-8730-2815
} 
Aspectos facilitadores e dificultadores do trabalho do enfermeiro em cargos gerenciais no... $\mid 2$

family interaction; work unit management; management group implementation and communication. In contrast, the hindering aspects are related to organizational structure, infrastructure management and people management. Descriptors: Nursing; Organization and administration; Public hospitals; Hospital administration

Resumen: Objetivo: identificar aspectos que facilitan y dificultan el trabajo del enfermero en puestos directivos en un hospital público de enseñanza. Método: estudio descriptivo con enfoque cualitativo, desarrollado en un hospital público terciario, utilizando la Técnica de Incidentes Críticos. Muestra compuesta por 15 enfermeros en puestos directivos durante al menos un año. Resultados: se obtuvieron 42 situaciones, el $66.6 \%$ de referencias negativas; 57 comportamientos presentaron $84.2 \%$ de referencias positivas y 74 consecuencias en las que se observó el $58.1 \%$ de referencias negativas. Conclusión: los aspectos que facilitan el trabajo del enfermero en puestos gerenciales son situaciones / comportamientos / consecuencias relacionadas con la interacción equipo / paciente / familia; gestión de unidades de trabajo; implementación del grupo de gestión y comunicación. Por el contrario, los aspectos difíciles están relacionados con la estructura organizativa, la gestión de la infraestructura y la gestión de personas.

Descriptores: Enfermería; Organización y Administración; Hospitales Públicos; Administración Hospitalaria

\section{Introdução}

A reconfiguração do papel dos hospitais na perspectiva da construção de redes de atenção à saúde, repercute na organização do trabalho de Enfermagem, na qual, cabe ao enfermeiro utilizar conhecimentos pertinentes às práticas do cuidado, realizar a assistência direta a usuários que estejam em maior grau de dependência de cuidados, além do gerenciamento dos recursos e das relações no trabalho, como planejamento, avaliação e execução dos serviços necessários à prática de Enfermagem..$^{1-2}$

Esse olhar aproxima-se da concepção atual de redes de atenção à saúde, conceituadas como sistema integrado de saúde que envolve relações singulares entre profissionais e usuários, em um continuum de serviços prestados à população, compreendendo as estruturas operacional, ambulatorial e hospitalar nos níveis primário, secundário e terciário. ${ }^{3-5}$ Os enfermeiros precisam apropriar-se das mudanças da sociedade contemporânea, desprender-se dos paradigmas clássicos da gerência fragmentada em tarefas e decisões centralizadas, tendo como base a atenção integral à saúde. ${ }^{6}$ Entende-se que esse processo dinâmico de trabalho do enfermeiro contribui para o enfrentamento das dificuldades no que diz respeito ao exercício desse trabalho na qualidade de gerente de hospital público de ensino, em especial, no tocante à comunicação, 
3 | Silva NM, Mininel VA, Henriques SH, Limongelli AMA, Pereira AP, Chaves LDP

relações de poder e hierarquia, gestão de pessoas e de recursos materiais, dentre outros dificultadores.

A relevância da temática e a carência da produção científica específica sobre o trabalho do enfermeiro em cargos gerenciais, após levantamento bibliográfico de publicações científicas, no período 2000-2014, na base de dados LILACS, com os descritores “enfermagem”, “organização” e “administração”, bem como a reconfiguração do papel do hospital na perspectiva das redes de atenção, justificaram a realização desta pesquisa. Entende-se que os resultados podem contribuir no sentido de apreender sobre os aspectos que favorecem e dificultam o trabalho do enfermeiro gerente, produzindo conhecimentos que subsidiem a assistência e a gerência em hospitais.

Frente o exposto e diante do problema de pesquisa levantado, questiona-se: "Quais são os aspectos facilitadores e dificultadores do trabalho do enfermeiro em cargo gerencial em hospital público de ensino?”. Nesse sentido, o estudo teve como objetivo identificar os aspectos que facilitam e dificultam o trabalho do enfermeiro em cargos gerenciais em um hospital público de ensino.

\section{Método}

Trata-se de um estudo descritivo, de abordagem qualitativa. Utilizou-se a Técnica do Incidente Crítico (TIC), que permite a identificação de percepções e atitudes dos participantes vivenciadas no trabalho e pertinentes ao objeto de investigação. ${ }^{7-8}$

Um incidente, para ser crítico, ocorre quando é possível estabelecer claramente a tríade situação, comportamento e consequência. A situação vivenciada ou observada é entendida como um fato cuja intenção ou propósito do ato seja claro a ponto de desencadear um respectivo comportamento diante do fato e as consequências são suficientemente definidas como os efeitos produzidos. ${ }^{9}$

Esta pesquisa foi desenvolvida em instituição hospitalar pública, referência no 
Aspectos facilitadores e dificultadores do trabalho do enfermeiro em cargos gerenciais no... $\mid 4$

atendimento a urgências e emergências. Conta com 171 leitos de internação e está localizada em município da região nordeste do Estado de São Paulo, Brasil.

Os participantes do estudo foram 15 enfermeiros em cargos gerenciais de unidades de internação adulto e pediátrica, pois o hospital presta atendimento, em maior escala, para esses usuários. Foram critérios de seleção para participar do estudo, ser enfermeiro e atuar em cargo gerencial na unidade há, no mínimo, um ano, pois, interpreta-se que esse tempo confere ao profissional a experiência de inserção em atividades gerenciais da unidade. Potencialmente, 18 enfermeiros atenderam aos critérios, mas três enfermeiros se recusaram a participar da pesquisa. Assim, o grupo do estudo foi composto por 15 participantes.

Para a coleta de dados, optou-se por utilizar a entrevista semiestruturada, guiada por um roteiro submetido à validação de face e de conteúdo por três peritos em metodologia científica e em temática. Posteriormente, foi realizado teste piloto com três enfermeiros que ocupavam cargo gerencial em unidades distintas daquelas selecionadas.

O roteiro era composto de duas partes: uma relativa à caracterização sociodemográfica e profissional e outra contendo questões abertas para relato de situações vivenciadas e/ou observadas, facilitadoras ou dificultadoras do trabalho do enfermeiro em cargo gerencial. As entrevistas foram realizadas individualmente por uma auxiliar de campo, uma enfermeira, aluna de pós-graduação, nível mestrado, que foi capacitada previamente para realizá-las. Justifica-se a utilização de auxiliar de campo pelo fato de a pesquisadora responsável ser membro do staff do hospital, pois tal situação poderia comprometer as respostas. Estas foram agendadas conforme a disponibilidade dos participantes.

Os dados foram coletados no período de julho de 2014 a janeiro de 2015. Para garantir a privacidade e o sigilo dos participantes, foram utilizados, para identificá-los, os códigos E1, E2, E3 e, assim, sucessivamente, sendo a letra E equivalente à entrevista e, o numeral, à ordem sequencial de realização. As entrevistas tiveram duração média de 40 minutos, foram gravadas, 
5 | Silva NM, Mininel VA, Henriques SH, Limongelli AMA, Pereira AP, Chaves LDP

transcritas e submetidas à extração dos Incidentes Críticos (IC), identificando situações, comportamentos e consequências, ${ }^{8}$ Nos IC relatados, foram mencionadas referências positivas ou negativas, levando-se em conta o julgamento do próprio participante, entendidas como aspectos facilitadores ou dificultadores do trabalho do enfermeiro em cargos gerenciais.

A identificação e o agrupamento das situações, comportamentos e consequências possibilitaram a categorização dos dados por semelhança de conteúdo. Nessa etapa, a utilização de fundamentos teóricos favoreceu a construção de categorias adequadas ao objeto de estudo. $\mathrm{Na}$ análise, foi utilizada estatística descritiva, que permitiu a descrição e síntese dos dados relativos às situações, comportamentos e consequências, ${ }^{9}$ tendo sido quantificados cada um dos elementos que compõem a tríade que caracteriza o IC. Posteriormente, os relatos foram analisados utilizando-se a Análise de Conteúdo. ${ }^{10}$

A pesquisa foi desenvolvida de modo a garantir o cumprimento dos preceitos da Resolução n 466/2012 sobre pesquisa envolvendo seres humanos, sendo aprovada pelo Comitê de Ética e Pesquisa da Escola de Enfermagem de Ribeirão Preto com o CAAE: 19860013.7.0000.5393, de 30 de outubro de 2013. Os participantes da pesquisa e os juízes receberam o Termo de Consentimento Livre e Esclarecido (TCLE).

\section{Resultados}

Em relação à faixa etária dos 15 enfermeiros em cargos gerenciais, obteve-se a idade mínima de 34 anos e máxima de 60 anos, predominando os indivíduos com idades acima de 44 anos, representando oito $(53,3 \%)$ dos participantes. Com relação ao sexo, predominam profissionais do sexo feminino representados por 13 (86,7\%) dos participantes. Em relação ao tempo de atuação em cargo gerencial na instituição, a maior parte 10 (66,6\%) dos enfermeiros atua entre 17 e 21 anos. Quanto ao tempo de formação, 10 (66,6\%) tinham entre 13 e 19 anos de formados e $5(33,4 \%)$ tinham acima de 20 anos de formação. 
Aspectos facilitadores e dificultadores do trabalho do enfermeiro em cargos gerenciais no... $\mid 6$

Os dados obtidos permitiram identificar incidentes críticos com 42 situações, sendo 14 positivas e 28 negativas.

As situações identificadas nos incidentes críticos foram agrupadas, criando as categorias: “estrutura organizacional”, “interação: equipe/paciente/familiar”, “gestão de infraestrutura”, “gestão de pessoas”.

Destaca-se que, dentre as situações, houve predomínio de referências negativas, totalizando $28(66,6 \%)$, ou seja, aspectos que dificultam o do trabalho do enfermeiro em cargos gerenciais. As situações identificadas estão apresentadas na Tabela 1.

Tabela 1 - Distribuição das categorias de situações extraídas dos incidentes críticos referentes ao trabalho gerencial do enfermeiro, de um hospital público, segundo referência positiva ou negativa. Ribeirão Preto, SP, 2015.

\begin{tabular}{lcccccc}
\hline \hline \multirow{2}{*}{ Categorias de situação } & \multicolumn{2}{c}{ Positiva } & \multicolumn{2}{c}{ Negativa } & \multicolumn{2}{c}{ Total } \\
& no & $\mathbf{\%}$ & no & \% & no & \% \\
\hline \hline Estrutura organizacional & 6 & 42,9 & 8 & 57,1 & 14 & 100 \\
Interação: equipe/paciente/familiar & 8 & 72,7 & 3 & 27,3 & 11 & 100 \\
Gestão de infraestrutura & 0 & 0 & 9 & 100 & 9 & 100 \\
Gestão de pessoas & 0 & 0 & 8 & 100 & 8 & 100 \\
Total & 14 & 33,4 & 28 & 66,6 & 42 & 100 \\
\hline \hline
\end{tabular}

A categoria de situação "Estrutura organizacional” refere-se às mudanças no modelo gerencial do hospital com a adesão ao modelo de gestão compartilhada. Percebe-se que existiram lacunas no processo de mudança do modelo de gestão caracterizadas pela falta de clareza na implantação do grupo gestor. Nessa categoria, somaram-se 57,1\% de referências negativas.

[...] quando houve a implantação do sistema de gestão compartilhada, tudo foi muito oprimido, não tínhamos autonomia sobre o serviço. Isso foi muito negativo, me deixou muito triste. Não tivemos apoio administrativo. (E1)

Embora prevaleçam referências negativas, as referências positivas alcançaram $42,9 \%$.

[...] tirei uma lição disso: nós, chefes, precisamos acreditar na força que temos porque, se estamos nesse cargo, não é à toa! Temos merecimento de 
7 | Silva NM, Mininel VA, Henriques SH, Limongelli AMA, Pereira AP, Chaves LDP

alguma forma ou porque exercemos a liderança ou porque queremos trabalhar. Fico muito contente com isso. (E14)

A categoria de situação "Interação: equipe/paciente/família” compila fatos relativos à relação interpessoal, de diálogo, mediação de conflitos entre equipes, familiares e usuários. Houve predomínio de referências positivas $(72,7 \%)$, evidenciando as ações de interlocução e mediação frequentes no cotidiano de trabalho do enfermeiro em cargo gerencial.

[...] tinha um paciente que ficou gravemente queimado, $90 \%$ de queimadura. Conforme ele foi melhorando, as equipes que estavam atendendo foram dando alta. Chegou em um momento que parecia que o paciente era da equipe de Enfermagem, somente. Neste momento, precisei solicitar uma reunião com os responsáveis pela cirurgia, plástica, vascular, nutrição e outros. Na reunião, deixei claro que todos éramos responsáveis pelo paciente [...] fiquei muito feliz com essa interação. (E13)

A fala que segue exemplifica a referência negativa nessa categoria de situações.

[...] tem várias situaçôes de gerenciamento, tipo, chega o familiar no balcão de atendimento e quer falar somente com o médico. Eu, mesmo sabendo que ele está ocupado, chamei. Ele não veio! [...] mesmo que eu tenha chamado o médico e tudo, mas quem levou a pior fui eu! Tem médico que fala: "Não vou conversar não", aí eu tenho que fazer o jogo de cintura entre o médico e a família. Isso me frustra. (E1)

A categoria de situações "Gestão de infraestrutura" agrupa questões vinculadas com o bloqueio de leitos devido à falta de infraestrutura e serviço de manutenção de equipamentos lento. Nesse sentido, a morosidade na gestão de infraestrutura emerge como aspecto dificultador do trabalho do enfermeiro gerente.

[...] acho que o problema maior é que nós não trabalhamos sozinhos, sempre dependemos dos outros. Eu sei que, se quebra um material, tem que ir para outro setor para arrumar; isso dificulta ter um resultado mais rápido nas ações [...] a manutenção de um equipamento aqui é muito demorada. (E15)

A categoria de situações "Gestão de Pessoas" agrega dados relacionados à redução de pessoal e absenteísmo. Essa categoria faz alusão apenas a referências negativas (100\%). 
Aspectos facilitadores e dificultadores do trabalho do enfermeiro em cargos gerenciais no... $\mid 8$

[...] todos os hospitais estão vivenciando e aqui eu vivencio todos os dias, falta de pessoal. Eu deixei de encaminhar um paciente para o exame porque faltaram funcionários nesse dia. (E1)

$\mathrm{Na}$ Tabela 2, são apresentados os 57 comportamentos, dos quais 48 foram positivos e nove, negativos. Os comportamentos foram classificados em três categorias: "Gerenciar a unidade de trabalho”, “Comunicar-se”, “Questionar a implantação do Grupo Gestor”. Cabe ressaltar que o número de comportamentos excedeu os números de situações, indicando que, para cada situação, pode existir mais de uma ação.

Tabela 2 - Distribuição das categorias de comportamentos extraídos dos incidentes críticos referentes ao trabalho gerencial do enfermeiro, de um hospital público, segundo referência positiva ou negativa. Ribeirão Preto, SP, 2015.

\begin{tabular}{lcccccc}
\hline \multirow{2}{*}{ Categorias de comportamentos } & \multicolumn{3}{c}{ Positiva } & \multicolumn{2}{c}{ Negativa } & \multicolumn{2}{c}{ Total } \\
\cline { 2 - 7 } & $\mathbf{n} \mathbf{0}$ & $\mathbf{\%}$ & $\mathbf{n}^{\mathbf{0}}$ & $\mathbf{\%}$ & $\mathbf{n}^{\mathbf{0}}$ & $\mathbf{\%}$ \\
\hline \hline Gerenciar a unidade de trabalho & 31 & 86,1 & 5 & 13,9 & 36 & 100 \\
Comunicar-se & 12 & 75,0 & 4 & 25,0 & 16 & 100 \\
Questionar a implantação do Grupo Gestor & 5 & 100 & 0 & 0 & 5 & 100 \\
Total & 48 & 84,2 & 9 & 17,8 & 57 & 100 \\
\hline \hline
\end{tabular}

A categoria de comportamentos - "Gerenciar a unidade de trabalho" relaciona-se às adequações do consumo diante da escassez de material e ajustes na escala mensal decorrentes do absenteísmo. Houve predomínio de referências positivas $(86,1 \%)$.

[...] se for grave mesmo, a regulação não quer nem saber, o médico contratado não quer nem saber, a regulação vai mandar, em vaga zero. $O$ que eu fiz?! Abri o computador, vi onde tinham leitos vagos e verifiquei quais pacientes estavam internados e que tinham condições de serem transferidos. (E2)

Embora em menor quantidade, há referências negativas (13,9\%) dessa categoria.

[...] faltaram funcionários nesse dia. Eu tive que priorizar funcionário para acompanhar a realização de exame ou ficar escalado na enfermaria. Eu optei por deixar o exame para depois, e isso é ruim. (E1)

A categoria de comportamentos "Comunicar-se" diz respeito à comunicação, interação 
9 | Silva NM, Mininel VA, Henriques SH, Limongelli AMA, Pereira AP, Chaves LDP

entre as equipes de trabalho, familiares e usuários, para a organização de atividades gerenciais e/ou assistenciais. Essa categoria teve predomínio de referências positivas (75\%).

[...] eu trouxe algumas coisas que eu fazia no campus, procurei trabalhar mais no sentido de conversar com o grupo e mostrar que isso eu posso ou isso eu não posso. E, dessa forma, eu consegui propor algumas mudanças [...] pelo menos, a minha taxa de absenteísmo diminuiu. (E12)

As referências negativas nessa categoria são menos frequentes (25\%), como pode ser

[...] o número de pessoas da equipe é reduzido, então, é angustiante porque o tempo para dialogar com o paciente e estabelecer vínculo é pequeno. (E12)

A categoria de comportamentos "Questionar a implantação do Grupo Gestor" diz respeito ao posicionamento questionador do enfermeiro frente à falta de autonomia instalada com a mudança do modelo de gestão e como esse posicionamento fortaleceu o grupo, diminuindo a vulnerabilidade profissional. A categoria teve somente referências positivas.

[...] apoiei o meu colega e o incentivei a pensar no todo, no serviço, nos funcionários competentes, nos pacientes, e a tomar decisões justas sem a motivação emocional que estávamos vivendo na época [...] os auxiliares e técnicos perceberam que ser chefe não é fácil, temos merecimento de alguma forma. (E3)

A Tabela 3 apresenta as 74 consequências identificadas, sendo 31 com referências positivas e 43 com referências negativas, apresentados em duas categorias: "Sentimentos", “Assistência prestada ao paciente”.

Tabela 3 - Distribuição das categorias de consequências extraídas dos incidentes críticos, referentes ao trabalho gerencial do enfermeiro, de um hospital público, segundo referência positiva ou negativa. Ribeirão Preto, SP, 2015.

\begin{tabular}{lccccccc}
\hline \multirow{2}{*}{ Categorias de consequências } & \multicolumn{2}{c}{ Positiva } & \multicolumn{2}{c}{ Negativa } & \multicolumn{2}{c}{ Total } \\
\cline { 2 - 8 } & $\mathbf{n} \mathbf{n}^{\mathbf{0}}$ & $\mathbf{\%}$ & $\mathbf{n} \mathbf{0}$ & $\mathbf{\%}$ & $\mathbf{n}^{\mathbf{0}}$ & $\mathbf{\%}$ \\
\hline \hline Sentimentos & 25 & 41,7 & 35 & 58,3 & 60 & 100 \\
Assistência prestada ao paciente & 6 & 42,9 & 8 & 57,1 & 14 & 100 \\
Total & 31 & 41,9 & 43 & 58,1 & 74 & 100 \\
\hline \hline
\end{tabular}

As consequências compõem-se do desfecho relacionado às ações dos enfermeiros em 
Aspectos facilitadores e dificultadores do trabalho do enfermeiro em cargos gerenciais no... | 10

cargos gerenciais frente às situações vivenciadas. É importante destacar que, nos incidentes críticos extraídos, embora os comportamentos frente às situações fossem predominantemente positivos, as consequências surgiram com maior frequência negativa.

A categoria de consequências "Sentimentos" reúne aspectos relativos a emoções, demonstração de afeto e tristeza. Cabe destacar que dizem respeito a um conjunto de questões que não estão necessariamente na governabilidade do enfermeiro, mas que perpassam o seu cotidiano e podem ser decorrentes de uma somatória de diferentes situações e comportamentos anteriormente apresentados. Houve predomínio de referências negativas (58,3\%).

[...] a situação foi gritante, os gestores demoraram a dar apoio, a administração demorou a tomar decisões e eu, como chefe, tive uma certa angústia. Toda equipe e eu nos sentimos impotentes, sozinhos durante três anos. (E9)

Apesar de menos frequente, há referências positivas (41,7\%) dessa categoria.

[...] o que tem de bom no gerenciamento de Enfermagem é que você pode fazer! Pode correr atrás, pode fazer acontecer! Só não faz quem não quer, quem está desmotivado. Eu faço, fiz e sempre fico muito satisfeita com isso; o paciente foi cuidado por todos e teve alta. (E13)

A categoria de consequências "Assistência prestada ao paciente" refere-se a aspectos relativos ao cuidado prestado no tocante ao controle de infecção hospitalar, segurança do paciente, infraestrutura e absenteísmo. Essa categoria teve predomínio de referências negativas (57,1\%).

[...] tinha uma goteira na enfermaria e foi muito demorado conseguir com que a manutenção solucionasse o problema. Tivemos que fazer vários relatórios [...] porque o maior medo que tinha era que aquela água fosse contaminada. (E5)

A fala que segue ilustra a referência positiva $(42,9 \%)$ dessa categoria.

[...] ainda falta muita coisa, mas, pelo menos, a taxa de absenteísmo diminuiu; trabalhar a confecção de escala de outra maneira, acho que isso 
11 | Silva NM, Mininel VA, Henriques SH, Limongelli AMA, Pereira AP, Chaves LDP

facilita. Você tentar negociar trocas e eu sei que as normas existem, elas precisam para manter a organização do serviço, mas tem coisas que podem ser resolvidas individualmente. (E6)

A síntese dos resultados que emergiram pode ser constatada na figura 1, a seguir.

\section{Situações}

$14(33,3 \%)$ positivas

$28(66,7 \%)$ negativas

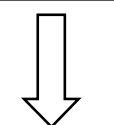

\section{Categorias}

-Estrutura organizacional

-Interação: Equipe/

Paciente/Familiar

-Gestão de infraestrutura

-Gestão de Pessoas

\section{Comportamentos}

$48(84,2 \%)$ positivos

Nove $(15,8 \%)$ negativos

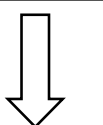

Categorias

- Gerenciar a unidade de trabalho

- Comunicar-se

- Questionar a implantação

de grupo gestor

\section{Consequências}

$31(41,9 \%)$ positivas

$43(58,1 \%)$ negativas

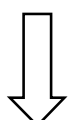

Categorias

- Sentimentos

- Assistência prestada

ao paciente

Figura 1 - Representação das situações, comportamentos e consequências e suas respectivas categorias, a partir dos relatos dos enfermeiros com cargos gerenciais da Unidade de Emergência do HCFMRP. Ribeirão Preto, SP, 2015.

\section{Discussão}

As situações da categoria "Estrutura organizacional” tiveram predomínio de referências negativas e pode evidenciar que, no processo de mudança no modelo gerencial na instituição, existiu lapso de implantação relativo à comunicação efetiva e à horizontalidade nos processos decisórios. Nesse sentido, depreende-se que tal processo, muito provavelmente, não considerou, em sua implantação, pressupostos que o fundamentam. Algumas estruturas organizacionais implicam determinado modelo de gestão, conformando, assim, distribuição de poder, decisão e comunicação caracterizados pelas ações participativas e descentralizadas nas quais as decisões perpassam por um processo multidisciplinar. ${ }^{11-12}$

As referências positivas na categoria "Estrutura organizacional” centraram-se na 
articulação profissional do enfermeiro em cargo gerencial com a equipe multiprofissional, facilitando a atuação e a parceria destes no cotidiano do trabalho na instituição. A parceria profissional e o fortalecimento das relações interpessoais, tanto entre os trabalhadores dos diferentes setores como também entre a equipe gestora da unidade hospitalar, são importantes para a gestão da equipe de Enfermagem e gestão do cuidado. ${ }^{13}$

As situações da categoria "Interação: equipe/paciente/família” tiveram predomínio de referências positivas, ou seja, é um aspecto que facilita o trabalho do enfermeiro em cargos gerenciais, evidenciando sua potencialidade para articular interlocução e relações.

A Política Nacional de Humanização aponta a gestão participativa como temas transversais para a construção em equipe. Assim, questões relacionadas à depreciação dos vínculos, precarização das condições de trabalho e privação dos direitos dos trabalhadores vão em sentido oposto a esses pressupostos. ${ }^{14-15}$

O atendimento a usuários de alta complexidade pressupõe ações interativas entre diversas categorias profissionais que, muitas vezes, podem resultar em conflito, por se tratar de instituição de atendimento de alta complexidade cujas necessidades são reais e imperativas. Nesse sentido, é preciso considerar peculiaridades individuais, profissionais e de contexto na construção de relações. ${ }^{16} \mathrm{O}$ trabalho do enfermeiro em cargos gerenciais envolve, entre outros, a interlocução e a mediação de situações de tensão entre trabalhadores, pacientes e familiares, a fim de viabilizar ambiente de trabalho propício, ${ }^{17}$ ratificando a percepção dos participantes sobre os aspectos dessa categoria "Interação: equipe/paciente/família” serem facilitadores do trabalho.

As situações da categoria "Gestão de infraestrutura" concentram apenas referências negativas, ou seja, foi considerada como aspecto dificultador para o trabalho do enfermeiro na gerência. Cabe destacar que a assistência prestada está diretamente ligada aos recursos materiais e de infraestrutura das instituições hospitalares. Assim, torna-se incontestável a 
13 | Silva NM, Mininel VA, Henriques SH, Limongelli AMA, Pereira AP, Chaves LDP

relevância da atuação do enfermeiro em cargo gerencial relacionado a esses recursos.

A escassez de recursos físicos, materiais e de pessoal qualificado mostra-se como aspecto dificultador para a atuação do enfermeiro, podendo incorrer na interrupção de procedimentos, bem como na compra de materiais e equipamentos realizada por profissionais que desconhecem as especificações adequadas que podem interferir na qualidade da assistência. ${ }^{18-19}$ Os profissionais de saúde de hospitais públicos de atendimento às urgências e emergências, de maneira geral, lidam com precárias condições de trabalho relacionadas à estrutura física, insuficiência de profissionais e falta de leitos, questões que impõem limitações alheias à vontade e à competência profissional. ${ }^{20}$

Destaca-se a relevância do gerenciamento de materiais, por tratar-se de produtos que são utilizados na prestação de assistência, de maneira direta, ao usuário, cujas dificuldades atuais estão diretamente relacionadas ao escasso montante financeiro destinado à saúde. ${ }^{20}$ As questões de infraestrutura dizem respeito a componentes do serviço de saúde estruturantes para a prática assistencial e gerencial, incluindo estrutura física, equipamentos, dentre outros. Entretanto, muitas vezes, a resolução desses problemas não está na governabilidade do enfermeiro.

As situações da categoria "Gestão de pessoas" tiveram apenas referências negativas. Trata-se de resultado muito preocupante, uma vez que esta é uma atividade precípua de profissionais em cargos gerenciais e, além disso, sem pessoas, o cuidado não se concretiza. Sabe-se que o quantitativo inadequado de trabalhadores de Enfermagem repercute diretamente no aumento da taxa de absenteísmo como reflexo da sobrecarga de atividades e insatisfação profissional. $^{13,21}$

Os comportamentos agrupados na categoria "Gerenciar a unidade de trabalho" foram considerados facilitadores do trabalho do enfermeiro gerente. $\mathrm{Na}$ organização dos serviços de atenção hospitalar, fazem-se necessários investimentos tecnológicos referentes a materiais e equipamentos e infraestrutura, para viabilizar melhor acesso aos usuários, bem como à adequação de pessoal qualificado para o atendimento aos agravos à saúde. ${ }^{19}$ Dentre as 
Aspectos facilitadores e dificultadores do trabalho do enfermeiro em cargos gerenciais no... | 14

atribuições gerenciais dos enfermeiros, está a previsão de pessoal, controle de recursos materiais e resolução de conflitos. Nesse contexto, o enfermeiro responsável por gerenciar esses recursos assume a responsabilidade de viabilizar e facilitar a assistência de Enfermagem na instituição. ${ }^{18}$

Os comportamentos da categoria "Comunicar-se" foi apontada como aspecto facilitador do trabalho do enfermeiro na gerência e, em relação a este aspecto, entende-se que a comunicação perpassa as atividades cotidianas de planejamento e organização do trabalho e, quando favorecida, pode ser um potencializador dos processos e relações. Tal ação caracterizase como comunicação efetiva, tendo em vista a inclusão de comportamento assertivo em transmitir, receber e interpretar informações de forma clara e respeitosa. O diálogo entre os trabalhadores, gestores e usuários é uma importante estratégia para desempenhar as atividades do cuidado de Enfermagem, uma vez que o diálogo constrói uma relação de integração de cultura e troca de experiências. ${ }^{22-23}$

Os comportamentos da categoria “Questionar a implantação do Grupo Gestor” foram considerados como elemento facilitador, enquanto forma de posicionamento dos enfermeiros em cargos gerenciais, diante do processo de flexibilização do modelo de gestão na instituição e, nesse sentido, cabe esclarecer que não se trata de resistência à mudança, mas à forma de operar as mudanças que foi adotada. A gestão participativa requer a descentralização e o envolvimento efetivo dos profissionais nas discussões e tomada de decisões de forma democrática. Traz a vantagem de elevar a legitimidade das decisões, sendo elas assertivas ou não. ${ }^{24}$

As consequências agrupadas na categoria "Sentimentos" concentram referências negativas e foi considerada como aspecto dificultador, visto que tais questões não estão apenas sob o controle do enfermeiro em cargo gerencial. A atividade laboral é considerada fonte de satisfação e prazer, porém, à medida que ocorrem falhas entre as expectativas do trabalhador e a realidade imposta pela gerência de trabalho, pode implicar sofrimento, ocasionando, entre 
15 | Silva NM, Mininel VA, Henriques SH, Limongelli AMA, Pereira AP, Chaves LDP

outros prejuízos, o adoecimento dos trabalhadores. ${ }^{24}$ No campo da saúde, a efetivação do trabalho não decorre exclusivamente da competência e do compromisso do trabalhador. As condições laborais são fundamentais para o desenvolvimento de suas ações e, quando são precárias, sujeitam o profissional ao estresse e ao sofrimento. ${ }^{21}$

Apesar do predomínio de referências negativas na categoria de consequências "Sentimentos", foi possível perceber que enfermeiros em cargos gerenciais apresentam, também, satisfação e contentamento, quando conseguem oportunidades em apontar as necessidades de ajustes no trabalho. Nesse sentido, percebe-se que a tristeza e a angústia, relatadas pelos participantes do estudo, são minimizadas pela interação com a equipe, a família e a satisfação em assistir o usuário.

As consequências agrupadas na categoria "Assistência prestada ao paciente" evidenciaram a preocupação acerca das questões que envolvem o cuidado dispensado ao usuário, sinalizando a compreensão da relação estreita entre gerenciamento e a assistência, pois refere o cuidado ao usuário como motivo maior para a mobilização de diferentes recursos. A interação entre as equipes e a autonomia profissional contribui para o desenvolvimento das habilidades essenciais para o cuidado, trazendo, assim, resultados positivos na recuperação dos pacientes, sendo considerados fatores facilitadores para a gestão de qualidade da assistência. ${ }^{13}$ Os aspectos dificultadores na gestão da qualidade da assistência estão relacionados ao número deficiente de profissionais diante da demanda cada vez mais complexa dos pacientes, da infraestrutura e dos recursos materiais deficitários nas organizações públicas de saúde. ${ }^{13}$

\section{Conclusão}

Concluiu-se que os aspectos facilitadores para o trabalho do enfermeiro em cargo gerencial são situações/comportamentos/consequências, com referências positivas, pertinentes à interação da equipe, usuário, família; destacando-se como fatores positivos a possibilidade de gerenciar a unidade de trabalho, questionar a implantação do grupo gestor e comunicar-se. Em 
Aspectos facilitadores e dificultadores do trabalho do enfermeiro em cargos gerenciais no... | 16

contrapartida, os aspectos dificultadores são situações/comportamentos/consequências com referências predominantemente negativas relacionadas às categorias estrutura organizacional, gestão de infraestrutura e gestão de pessoas.

Cabe ressaltar que, embora as situações tenham concentrado referências negativas, os comportamentos gerados foram reconhecidos pelos participantes como preponderantemente positivos, evidenciando o empenho e o esforço dos enfermeiros em cargos gerenciais para superar situações que dificultam seu trabalho e que, muitas vezes, envolvem agentes e/ou setores externos cuja governabilidade do enfermeiro em cargo gerencial é muito limitada.

Os resultados obtidos neste estudo contribuem para a temática gestão e administração hospitalar, particularmente para o gerenciamento de Enfermagem, no sentido de ratificar a complexidade e dificuldade para a implantação de um novo modelo de gestão, além de identificar as relações multidimensionais entre situação, comportamento e consequência que permeiam o cotidiano do trabalho do enfermeiro em cargo gerencial de um hospital público de urgência. Além disso, embora os resultados não sejam generalizáveis, entende-se como possíveis contribuições deste estudo os conhecimentos acerca do exercício de cargo gerencial para enfermeiros que atuam em distintos pontos de redes de atenção à saúde cujo trabalho envolve a singularidade das relações profissionais, assistenciais e de poder.

Esta pesquisa possui limitação no que se refere ao cenário de investigação, pois, no estudo, optou-se por ouvir enfermeiros em cargo de gerência de instituição hospitalar pública. Para pesquisas futuras, recomenda-se ampliar o estudo, a fim de abranger outras categorias profissionais com vistas à comparação e/ou generalização dos dados.

\section{Referências}

1. Lima RS, Lourenço EB, Rosado SR, Fava SMCL, Sanches RS, Dázio EMR. Representação da prática gerencial do enfermeiro na unidade de internação: perspectiva da equipe de enfermagem. Rev Gaúch Enferm [Internet]. 2016 mar [acesso em 2015 mar 01];37(1):e54422. Disponível em: http://www.scielo.br/pdf/rgenf/v37n1/0102-6933rgenf-37-1-1983-144720160154422.pdf doi:10.1590/1983-1447.2016.01.54422

2. Ferreira VHS, Teixeira VM, Giacomini MA, Alves LR, Gleriano JS, Chaves LDP, et al. Contribuições e 
17 | Silva NM, Mininel VA, Henriques SH, Limongelli AMA, Pereira AP, Chaves LDP

desafios do gerenciamento de enfermagem hospitalar: evidências científicas. Rev Gaúch Enferm [Internet]. 2019 [acesso em 2019 ago 01];40:e20180291. Disponível em: https://www.seer.ufrgs.br/RevistaGauchadeEnfermagem/article/view/93531/0 doi:https://doi.org/10.1590/1983-1447.2019.20180291

3. Mendes EV. As redes de atenção à saúde. Brasília (DF): Organização Pan-Americana da Saúde; 2011.

4. Mendes EV. Entrevista: a abordagem das condições crônicas pelo Sistema Único de Saúde. Ciênc Saúde Colet [Internet]. 2018 [acesso em 2019 ago 01];23(2):431-5. Disponível em: http://www.scielo.br/pdf/csc/v23n2/1413-8123-csc-23-02-0431.pdf _ doi:http://doi.org/10.1590/141381232018232.16152017

5. Fernandes MC, Silva LMS. Gerência do cuidado de enfermagem na estratégia saúde da família: revisão integrativa . Rev Rene [Internet]. 2013 [acesso em 2015 jun 01];14(2):438-47. Disponível em: http://www.periodicos.ufc.br/rene/article/view/3407/2644

6. Chaves LDP, Mininel VA, Silva JAM, Alves LR, Silva MF, Camelo SHH. Supervisão de enfermagem para a integralidade do cuidado. Rev Bras Enferm [Internet]. 2017 fev [acesso em 2019 ago 01];70(5):116570. Disponível em: http://www.scielo.br/pdf/reben/v70n5/pt_0034-7167-reben-70-05-1106.pdf doi:http://dx.doi.org/10.1590/0034-7167-2016-0491

7. Polit DF, Beck CT. Fundamentos de pesquisa em enfermagem: avaliação de evidências para a prática da enfermagem. 9ª ed. Porto Alegre: Artmed; 2019.

8. Flanagan JC. A técnica do incidente crítico. Arq Bras Psicol Apl [Internet]. 1973 [acesso em 2015 fev 02];25(2):99-141. Disponível em: http://bibliotecadigital.fgv.br/ojs/index.php/abpa/article/viewFile/16975/15786

9. Dela Coleta JA, Dela Coleta MF. A técnica de incidentes críticos: 30 anos de utilização no Brasil na psicologia, administração, saúde e educação. Taubaté: Cabral Editora e Livraria Universitária; 2004.

10. Bardin L. Análise de conteúdo. Lisboa: Edições 70; 2011.

11. Garcia AB, Maziero VG, Rocha FLR, Bernardes A, Gabriel CS. Influência da cultura organizacional na gestão participativa em organizações de saúde. Rev Pesqui Cuid Fundam [Internet]. 2015 abr-jun [acesso em 2015 nov 06];7(2):2615-27. Disponível em: https://www.redalyc.org/pdf/5057/505750946039.pdf doi: 10.9789/2175-5361.2015.v7i2.2615-2627

12. Rabenschlag LA, Lima SBS, Eberhardt TD, Kessler M, Soares RSA, Camponogara S. Gestão de qualidade na assistência de enfermagem em unidades de clínica cirúrgica. Rev Enferm UFSM [Internet] 2015 abr-jun [acesso em 2015 out 20];5(2):235-46. Disponível em: https://periodicos.ufsm.br/reufsm/article/view/15891/pdf doi: 10.5902/2179769215891

13. Lima Neto AV, Fernandes RL, Barbosa IML, Carvalho GRP, Nunes VMA. Relacionamento interpessoal entre a equipe de uma emergência hospitalar: um estudo qualitativo sob o olhar de enfermeiros. Enferm Rev [Internet]. 2015 jan-abr [acesso em 2015 maio 22];18(1):75-87. Disponível em: 
http://periodicos.pucminas.br/index.php/enfermagemrevista/article/view/9371/10328

14. Silva EM, Moreira MCN. Equipe de saúde: negociações e limites da autonomia, pertencimento e reconhecimento do outro. Ciênc Saúde Colet [Internet]. 2015 [acesso em 2015 out 27];20(10):3033-42. Disponível em: http://www.scielo.br/pdf/csc/v20n10/1413-8123-csc-20-10-3033.pdf doi: 10.1590/1413812320152010.20622014

15. Falcão KOM, Chagas MGR, Bezerra STF, Moura DJM, Cabral RL, Coelho MMF. Relações interpessoais entre profissionais de enfermagem de emergência hospitalar: proposta de intervenção. Rev Enferm UFPI [Internet]. 2014 out-dez [acesso em 2015 abr 04];3(4):95-100. Disponível em: http://www.ojs.ufpi.br/index.php/reufpi/article/view/1959/pdf

16. Camelo SHH. Competência profissional do enfermeiro para atuar em Unidade de Terapia Intensiva: uma revisão integrativa. Rev Latinoam Enferm [Internet]. 2012 jan-fev [acesso em 2015 jan 28];20(1):192200. Disponível em: http://www.scielo.br/pdf/rlae/v20n1/pt_25.pdf doi: 10.1590/S0104-11692012000100025

17. Oliveira MAN, Rosa DOS. Conflitos e dilemas éticos vivenciados pelo enfermeiro no cuidado perioperatório. Ciênc Cuid Saúde [Internet]. 2015 abr-jun [acesso em 2015 nov 08];14(2):1149-56. Disponível em: http://periodicos.uem.br/ojs/index.php/CiencCuidSaude/article/view/19423/14709 doi: 10.4025/cienccuidsaude.v14i2.19423

18. Lima PC, Sabino KCV, Gouveia MTO, Avelino FVSD, Fernandes MA. Fatores estressores e as estratégias de enfrentamento utilizadas por enfermeiros hospitalares: revisão. Investig Enferm [Internet]. 2015 jul-dez [acesso em 2016 jun 05];17(2):13-100. Disponível em: http://www.redalyc.org/articulo.oa?id=145239088006 doi: 10.11144/Javeriana.ie17-2.feea

19. Leal LA, Soares MI, Silva BR, Chaves LDP, Camelo SHH. Desafios para desenvolver competências no âmbito hospitalar. REME Rev Min Enferm [Internet]. 2018 [acesso em 2019 ago 01];22:e-1099. Disponível em: http://www.reme.org.br/artigo/detalhes/1250 doi: http://www.dx.doi.org/10.5935/1415-2762.20180042

20. Gil RB, Chaves LDP, Laus AM. Gerenciamento de recursos materiais com enfoque na queixa técnica. Rev Eletrônica Enferm [Internet]. 2015 jan-mar [acesso em 2015 dez 18];17(1):100-7. Disponível em: https://revistas.ufg.br/fen/article/view/27544/18951 doi: 10.5216/ree.v17i1.27544

21. Ferreira RC, Griep RH, Fonseca MJ, Rotenberg L.Abordagem multifatorial do absenteísmo por doença em trabalhadores de enfermagem. Rev Saúde Pública [Internet]. 2012 fev [acesso em 2015 mar 12];46(2):259-68. Disponível em: http://www.scielo.br/pdf/rsp/v46n2/3189.pdf doi: 10.1590/S0034-89102012005000018

22. Broca PV, Ferreira MA. Equipe de enfermagem e comunicação: contribuições para o cuidado de enfermagem. Rev Bras Enferm [Internet]. 2012 jan-fev [acesso em 2014 set 03];65(1):97-103. Disponível em: http://www.scielo.br/pdf/reben/v65n1/14.pdf doi: 10.1590/S0034-71672012000100014

23. Hayashida KY, Bernardes A, Maziero VG, Gabriel CS. A tomada de decisão da equipe de enfermagem após a revitalização do modelo compartilhado de gestão. Texto \& Contexto Enferm [Internet]. 2014 abr- 
jun [acesso em 2015 fev 01];23(2):286-93. Disponível em: http://www.scielo.br/pdf/tce/v23n2/pt_0104-0707tce-23-02-00286.pdf doi: 10.1590/0104-07072014001190013

24. Santos JLG, Prochnow AG, Silva DC, Silva RM, Leite JL, Erdmann AL. Prazer e sofrimento no exercício gerencial do enfermeiro no contexto hospitalar. Esc Anna Nery Rev Enferm [Internet]. 2013 jan-mar [acesso em 2015 maio 30];17(1):97-103. Disponível em: http://www.scielo.br/pdf/ean/v17n1/14.pdf doi: 10.1590/S1414-81452013000100014

\section{Autor correspondente}

Lucieli Dias Pedreschi Chaves

E-mail: dpchaves@eerp.usp.br

Endreço: Av. Banderantes, 3900 - Ribeirão Preto - SP

CEP: $14040-902$

\section{Contribuições de Autoria}

\section{1 -Nilce Mara da Silva}

Concepção/desenvolvimento do projeto, discussão dos dados e redação final do artigo

\section{2 - Vivian Aline Mininel}

Discussão dos dados, redação do artigo e revisão crítica

\section{3 - Silvia Helena Henriques}

Discussão dos dados, redação do artigo e revisão crítica

\section{4 - Ana Martha de Almeida Limongelli}

Discussão dos dados, redação do artigo e revisão crítica

\section{5 -Ana Paula Pereira}

Coleta/discussão dos dados e revisão crítica

\section{6 - Lucieli Dias Pedreschi Chaves}

Concepção/desenvolvimento do projeto, discussão dos dados e redação final do artigo

\section{Como citar este artigo}

Silva NM, Mininel VA, Henriques SH, Limongelli AMA, Pereira AP, Chaves LDP. Aspectos facilitadores e dificultadores do trabalho do enfermeiro em cargos gerenciais no âmbito hospitalar. Rev. Enferm. UFSM. 2020 [Acesso em: Anos Mês Dia]; vol.10 e8: 1-19. DOI:https://doi.org/10.5902/2179769233263 